\title{
Der zivilrechtliche Schutz vor sexueller Belästigung am Arbeitsplatz im chinesischen ZGB
}

\section{ZHAO Jin}

\section{Fragestellung}

Nach jüngeren Studien passiert es zurzeit in China nicht selten, dass ein Arbeitnehmer oder eine Arbeitnehmerin am Arbeitsplatz sexuell belästigt wird. Dies trifft insbesondere junge Arbeitnehmerinnen, die gerade ins Berufsleben eingestiegen sind. Auf Druck der Gesellschaft hin ist $\mathbb{1 0 1 0}$ ins Buch des Persönlichkeitsrechts des ZGB eingeführt worden. $\$ 1010$ ZGB lautet: Wird eine sexuelle Belästigung entgegen dem Willen einer anderen Person, etwa durch Worte, Texte, Bilder, körperliche Handlungen begangen, ist das Opfer berechtigt, nach dem Recht zu fordern, dass der Täter zivilrechtlich haftet. Institutionen, Unternehmen und Schulen haben angemessene Maßnahmen, wie Vorbeugungsmaßnahmen, Entgegennahme von Beschwerden, Untersuchungen und Sanktionen, zu ergreifen, sodass sexuelle Belästigungen, insbesondere durch Ausnutzung von Macht, vorbeugend verhindert werden. Im Vergleich zu $₫ 11$ Frauenschutzgesetz und $\$ 11$ der speziellen Verordnung über Arbeitsschutz der Arbeitnehmerinnen werden nicht nur Frauen, sondern alle natürlichen Personen vor sexueller Belästigung geschützt. Darunter fällt selbstverständlich der Schutz vor sexueller Belästigung am Arbeitsplatz. Unklar bleiben aber noch die Fragen, welches Verhalten am Arbeitsplatz als sexuelle Belästigung klassifiziert werden kann und wie der Arbeitgeber bei sexueller Belästigung haftet.

Dieser Beitrag gibt einen Überblick über die rechtswissenschaftliche Diskussion in Literatur und Rechtsprechung. Die zwei oben genannten Fragen werden erörtert und anschließend kritisch bewertet.

\section{Wann liegt eine sexuelle Belästigung am Arbeitsplatz vor?}

\section{Soziale Etikette als Beurteilungskriterium?}

Aus $₫ 1010$ S. 1 ZGB ergibt sich, dass die Worte „Entgegen dem Willen des anderen" das Kernstück sexueller Belästigung bilden. Das Vorliegen einer 
sexuellen Belästigung hängt somit davon ab, ob das in Frage stehende Verhalten gegen den Willen des Betroffenen verstößt. $\mathrm{Zu}$ der Bedeutung dieses elementaren Begriffs steht dessen wissenschaftliche Behandlung in einem Missverhältnis. In China sind wissenschaftliche Untersuchungen zu der Formulierung „Entgegen dem Willen des anderen“ selten.

Vor dem Inkrafttreten des ZGB beurteilte ein Teil der Rechtsprechung das Vorliegen sexueller Benachteiligung nach „allgemeiner sozialer Etikette". Zum Beispiel hat das Chengdu Wuhou Volksgericht das Vorliegen einer sexuellen Belästigung mit der Begründung anerkannt, dass das Verhalten des Beklagten von der allgemeinen sozialen Etikette abgewichen ist, indem der Beklagte mit einer Umarmung und Berührung nicht aufhörte, obwohl dies von der Klägerin erkennbar abgelehnt wurde. ${ }^{1}$

Die Frage, die sich nunmehr stellt, lautet: Eignet sich die allgemeine soziale Etikette als Beurteilungsmaßstab, um das Vorliegen einer sexuellen Belästigung zu klassifizieren? Dies setzt zunächst voraus, dass Einigkeit über die allgemeine soziale Etikette bei sexuellem Verhalten besteht, sodass Rechtsunsicherheit vermieden werden kann. In einer vielfältigen Gesellschaft ist es aber schwer zu beurteilen, welche bestimmten Verhaltensweise von der sozialen Etikette abweichen. Für manche Chinesen, die von der westlichen Kultur beeinflusst werden, ist eine freundliche Umarmung nur eine Form der Begrüßung. Andere halten diese für taktlos und unakzeptabel. Unterschiedlich ist ebenfalls die Bewertung einiger Verhaltensweisen, wie etwa Bemerkungen sexuellen Inhalts oder Verlangen nach privaten Fotos. Während manche solche Verhaltensweisen für scherzhaft oder lustig halten, werden solche Verhaltensweisen teilweise als Verstoß gegen die soziale Etikette angesehen. ${ }^{2}$ Diese Kontroversen werden eben-

1 Urteil des Volksgerichts Chengdu Wuhou im Rechtsstreit um die sexuelle Belästigung Xu gegen Liu, (2019), Chuan 0107 Min Chu Nr. 1407. Sachverhalt: Die Klägerin und der Beklagte arbeiteten zusammen in einer NGO. Eines Tages bot der Beklagte eine Umarmung an, die normalerweise als ein Ausdruck von Trösten in der NGO angesehen wird. Nach der ersten Umarmung umarmte der Beklagte die Klägerin fest. Da die Klägerin sich unwohl fühlte, schob sie den Beklagten weg. Doch der Beklagte legte seinen Arm um ihre Taille. Die Klägerin legte beim Volksgericht im Wuhou Distrikt in Chengdu Klage gegen den Beklagten ein, um Schadenersatz wegen sexueller Belästigung zu verlangen.

2 WU Fan (吴帆)/ ZHOU Ziyao (周子至), Der aktuelle Stand und Toleranz sexueller Belästigung im Bereich der sozialen Arbeit (社会工作职场性骚扰与容忍度研究), Journal of Chinese Women's Studies (妇女研究论丛), 2020, Nr. 5, S. 71, 76 ff. 
falls durch die jüngere Rechtsprechung bestätigt. ${ }^{3}$ Während mehrmalige SMS mit dem Inhalt „Vermisse dich“ und „Ich mag dich" vom Mittleren Volksgericht Zhongshan als sexuelle Belästigung eingestuft wurden, ${ }^{4}$ lehnte das Mittlere Volksgericht Nanjing das Vorliegen sexueller Belästigung ab, obwohl der Beklagte die Klägerin dazu nötigte, mit einem Kunden zu schlafen. ${ }^{5}$ Daraus kann man schließen, dass „soziale Etikette“ keinen objektiv nachprüfbaren Maßstab setzen kann. Ein weiteres Problem bei „sozialer Etikette“ besteht darin, dass es auf ein ideales Opferverhalten abstellt, was zu einer sekundären Verletzung des Opfers führen könnte.

\section{Unerwünscht als Kriterium?}

Unter dem Einfluss des US-amerikanischen Rechts wird von einem Teil der Literatur vorgeschlagen, den Begriff „unerwünscht (unwelcome)“ im chinesischen Recht einzuordnen, was eine große Rolle in der Beurteilung des Vorliegens einer sexuellen Belästigung im US-amerikanischen Recht spielt. ${ }^{6}$ Sodann soll „Entgegen dem Willen des anderen“ im Sinne von $\$ 1010$ ZGB als „unerwünscht (unwelcome)“ interpretiert werden. ${ }^{7}$ Dies spiegelt sich in der Anleitung für Prävention und Bekämpfung der sexuellen Belästigung ${ }^{8}$ wider. Diese Anleitung definiert sexuelle Belästigung als sexuelle unerlaubte Handlung im Deliktsrecht, wenn ein unerwünschtes, sexuell bestimmtes Verhalten entgegen dem Willen einer anderen Person begangen wird, durch das ein von Einschüchterungen, Anfeindungen, Er-

3 WANG Tianyu (王天玉), Logik in der Rechtsprechung über die verbale sexuelle Belästigung am Arbeitsplatz (言辞型职场性骚扰的司法裁判逻辑), Journal of Chinese Women's Studies (妇女研究论丛), 2020, Nr. 5, S. 60, 62.

4 Urteil des Mittleren Volksgerichts Zhongshan im Rechtsstreit über sexuelle Belästigung, (2016), Yue 20 Min Zhong Nr. 3456.

5 Urteil des Volksgerichts Suzhou Gongyeyuan im Rechtsstreit über sexuelle Belästigung, (2016), Su 0591 Min Zhong Nr. 9774.

6 Vgl. YANG Mao (杨茂), Die eigenständige Haftung des Arbeitgebers bei sexueller Belästigung am Arbeitsplatz (论职场性骚扰行为用人单位的独立责任), Qian Yan (前沿), 2011, Nr. 11, S. 93, 94.; LU Jiefeng (卢杰峰), Die Haftung des Arbeitgebers bei sexueller Belästigung am Arbeitsplatz (职场性骚扰的用人单位责任), Journal of Chinese Women's Studies (妇女研究论丛), 2020, Nr. 5, S. 87, 93; SHAN Chun (单 纯), Die Rechtsentwicklung über die sexuelle Belästigung am Arbeitsplatz in den USA (论美国反性骚扰的法治化进程), Journal of CUPL (中国政法大学学报), 2020, Nr. 4, S. $15 \mathrm{ff}$.

7 LU Jiefeng (Fn. 6), S. 92.

8 深圳市防治性骚扰行为指南,v. 15. 3. 2021, Shen Fu Tong (2021), Nr. 1. (深妇通 [2021]1 号). 
niedrigungen, Entwürdigungen oder Beleidigungen gekennzeichnetes Umfeld geschaffen wird. Außer Acht gelassen wird jedoch die genaue Bedeutung von „unerwünscht“. In diesem Zusammenhang ist es sinnvoll, auf die konkrete Bedeutung von „unerwünscht“ und den zugrundlegenden Grundgedanken im US-amerikanischen Recht einzugehen.

In den USA wird der Begriff „unerwünscht" vor allem von einer opferbezogenen Sichtweise geprägt. ${ }^{9}$ Dabei spielt es keine Rolle, ob der Betroffene sich in einer ausdrücklichen Weise gegen den angeblich Belästigenden wehrt. In Bezug auf Geschlechtsverkehr kann auf die Erwünschtheit nicht geschlossen werden, selbst wenn die betroffene Person dem Geschlechtsverkehr mit dem angeblich Belästigendem zugestimmt hat. So ließ sich in einem Fall das Opfer auf den Geschlechtsverkehr nur ein, weil es Angst vor beruflichen Nachteilen im Falle einer Ablehnung hatte. ${ }^{10}$ Dies folgt aus dem ursprünglichen Grundgedanken, dass hierarchische Geschlechterverhältnisse in der Arbeitswelt und dem Alltagsleben bestehen, innerhalb denen ein Geschlecht (Mann) über das andere Geschlecht (Frau) gestellt wird. Eine solche Geschlechterhierarchie spiegelt sich wiederum in der Realität sexueller Belästigungen wider. So sind Frauen als Opfer sexueller Belästigungen wesentlich häufiger betroffen als Männer. ${ }^{11}$ Dies trifft insbesondere auf berufstätige Frauen zu, deren Vorgesetzte Männer sind. In einem hierarchischen Verhältnis zwischen Vorgesetzten und Untergebenen ist es für den Untergebenen schwierig, die sexuelle Aufforderung des Vorgesetzten erfolgreich abzulehnen. Denn das Opfer muss mit möglichen beruflichen Nachteilen rechnen, die sich negativ auf den Lebensstandard des Opfers auswirken könnten. ${ }^{12}$ Ebenfalls spiegelt sich eine solche Ungleichheit in Geschlechterstereotypen wider. Werden Frauen als schüchtern oder passiv beim Geschlechtsverkehr angesehen, könnte ein "Nein“ auch als "Ja“ verstanden werden, obwohl das sexuelle Verhalten eigentlich nicht von der betroffenen Arbeitnehmerin erwünscht ist. ${ }^{13}$ Zur Lösung dieses Problems muss Frauen ein Recht auf Selbstbestimmung

9 Susanne Baer, Würde oder Gleichheit?, 1. Auflage, Baden-Baden 1995, S. 256.

10 Meritor Savings Bank, FSB v. Vinson, 477 U.S. 57, Supreme Court of the United States (June 19th, 1986).

11 Vgl. European Commission, Sexual Harassment at the Workplace in the European Union, Luxembourg 1999.

12 Catharine Mackinnon, Sexual harassment of working women, New Haven and London, Yale University Press, S. 39, 47.

13 Catharine Mackinnon (Fn. 12), S. 181. 
gewährt werden, sodass die Frauen als Opfer selbst definieren, was sexuelle Belästigung ist. ${ }^{14}$

Zusammenfassend ist zu sagen, dass „unerwünscht“ als Beurteilungskriterium besser ermöglicht, die durch Machtausübung begangene sexuelle Belästigung zu verhindern. Denn das Opfer braucht nicht nachzuweisen, dass es das in Rede stehende Verhalten ausdrücklich abgelehnt hat. Gerade aus diesem Grund wird der Begriff von „unerwünscht" auch in der EU bzw. in Deutschland ( $\$ 3$ Abs. 4 AGG) verwendet. Meines Erachtens könnte der Begriff „unerwünscht“ und seine konkrete Bedeutung ins chinesische Recht übernommen werden. Demnach soll die Person, die ein sexuelles Verhalten ausübt, mit einem solchen Verhalten aufhören, soweit die betroffene Person schweigt oder weggeht. Begeht sie die Handlung weiter, verstößt sie gegen den Willen der anderen Person i. S. v. $\$ 1010$ ZGB. Die Handlung stellt dann eine sexuelle Belästigung dar.

\section{Verletzung der Pflichten im Sinne von \$1010 S. 2 ZGB}

$\$ 1010$ S. 2 ZGB regelt, dass der Arbeitgeber angemessene Maßnahmen zu treffen hat, um die Begehung sexueller Belästigungen durch Ausnutzung etwa von Zugehörigkeitsverhältnissen zu verhindern oder zu unterbinden. $\mathrm{Zu}$ den Maßnahmen gehören präventive Maßnahmen, die Entgegennahme von Beschwerden, Untersuchungen und Sanktionen. Im Einzelfall bleibt es einem Arbeitnehmer aber unklar, welche konkreten Maßnahmen zur Verhinderung von sexueller Belästigung er von seinem Arbeitgeber fordern kann. Meines Erachtens könnten konkrete Maßnahmen, zu denen der Arbeitgeber nach US-amerikanischen und deutschen Regelungen verpflichtet ist, im Wege der Auslegung von chinesischen Gerichten entwickelt werden. Zur Prävention sexueller Belästigung sollte der Arbeitgeber durch betriebsinterne Richtlinien oder entsprechende Schulungen informieren, welches Verhalten als sexuelle Belästigung nicht tolerierbar ist und was Arbeitnehmer dagegen tun können. Weiter ist zu verlangen, dass für belästigte Beschäftigte Beschwerdewege eingerichtet werden und die Beschwerden in einer angemessenen Weise behandelt werden.

Zur Feststellung der Pflichtverletzung müssen aber die Umstände im chinesischen Arbeitsleben berücksichtigt werden. Unzweifelhaft liegt eine Pflichtverletzung vor, wenn der Geschäftsführer oder gesetzliche Vertreter des Arbeitgebers unerwünschte sexuelle Handlungen vornehmen. Wird

14 Catharine Mackinnon (Fn. 12), S. 181. 
der betroffene Arbeitnehmer durch Vorgesetzte oder Kollegen sexuell belästigt, liegt eine Pflichtverletzung des Arbeitgebers vor, wenn er die Pflichten i. S. v. $\$ 1010$ S. 2 ZGB nicht erfüllt hat. Die Pflicht zur Prävention soll jedoch nicht so streng sein wie im US-amerikanischen oder deutschen Recht. Gemäß $\$ 12$ AGG ist der Arbeitgeber verpflichtet, eine Beschwerdestelle für alle betroffenen Beschäftigten einzurichten. Daneben muss der Arbeitgeber alle betroffenen Beschäftigten durch Schulungen darüber informieren, welches Verhalten als sexuelle Belästigung klassifiziert wird und nicht tolerierbar ist. Eine solche Schulung bedarf aber professioneller Trainer und qualifizierte Fachkräfte, da es nicht einfach ist, über sexuelle Belästigung, die als ein sensibles Thema angesehen wird, in einer angemessenen Weise vor Publikum zu sprechen. Zurzeit stehen den Unternehmen in China nicht genügend Fachkräfte oder Trainer zur Verfügung. Daher ist eine große Anzahl chinesischer Arbeitgeber nicht in der Lage, solche Schulungsprogramme anzubieten. Vor diesem Hintergrund eignet es sich nicht, das Fehlen an der Schulung als Pflichtverletzung im chinesischen Kontext zu klassifizieren. Eine Pflichtverletzung liegt vor, wenn der Arbeitgeber keine angemessene Maßnahme ergriffen hat, obwohl sich der betroffene Arbeitnehmer schon bei dem Arbeitgeber über sexuelle Belästigung beschwert hat. Dies trifft insbesondere auf die Fälle zu, in denen der Arbeitgeber die Beschwerde ignoriert oder der Arbeitgeber das Opfer die Situation ertragen lässt, sodass der betroffene Arbeitnehmer wiederholt sexuell belästigt wird.

\section{Deliktsrechtlicher Anspruch des Arbeitnehmers gegenüber dem Arbeitgeber bei Belästigung durch Dritte}

Genauso wie das deutsche Recht bezweckt auch das chinesische Deliktsrecht den Schutz der Rechtsgüter einer Person. Wie oben dargelegt, wird der Schutz vor sexueller Belästigung ins Buch des Persönlichkeitsrechts eingeordnet. Daher ist davon auszugehen, dass sexuelle Belästigung als eine Verletzung des Persönlichkeitsrechts klassifiziert wird. Sodann greift zunächst $\$ 1165 \mathrm{ZGB}^{15}$ ein, wonach der Verletzte dem Geschädigten den erheblichen immateriellen Schaden ersetzen soll, der durch die Verlet-

$15 \$ 1165$ [Verschuldenshaftung; Verschuldensvermutung] Fügt ein Handelnder einem anderen durch eine schuldhafte Verletzung der zivilen Rechte und Interessen eine Schädigung zu, haftet er für die Verletzung von Rechten. Wird auf Grundlage von gesetzlichen Bestimmungen vermutet, dass das Verschulden beim Handelnden vorliegt, haftet er für die Verletzung von Rechten, wenn er nicht 
zung der persönlichen Rechte oder Interessen herbeigeführt wird. In der Praxis geht sexuelle Belästigung oftmals nicht vom Arbeitgeber selbst, sondern von Vorgesetzten des Opfers oder von gleichrangigen Kollegen aus. Nun soll die Rechtsfolge untersucht werden, wenn der Arbeitgeber die Pflicht im Sinne von $₫ 1010$ S. 2 ZGB verletzt und ein Arbeitnehmer sexuell belästigt wird. Sodann kommt zunächst ein Anspruch des Arbeitsnehmers aus Deliktsrecht in Betracht. Zugleich könnten die Regelungen aus dem Vertragsrecht und Arbeitsvertragsrecht anwendbar sein.

\section{Unterschiedliche Verschuldensvoraussetzungen je nach der Art der sexuellen Belästigung?}

In der Praxis geht sexuelle Belästigung am Arbeitsplatz meistens nicht vom Arbeitgeber selbst, sondern von Vorgesetzten des Opfers oder von gleichrangigen Kollegen aus. Dann stellt sich die Frage, wann eine deliktsrechtliche Ersatzpflicht den Arbeitgeber trifft. Dies kommt darauf an, wie das Handeln des belästigenden Vorgesetzten oder Kollegen dem Arbeitgeber zuzurechnen ist.

Teilweise wird vertreten, dass sich das Verschulden des Arbeitgebers an den Haftungsregeln des US-amerikanischen Rechts orientieren könne. ${ }^{16}$ Im US-amerikanischen Recht hängt die Haftung des Arbeitgebers von der Art der sexuellen Belästigung ab. Für „Quid pro quo harassment“-Belästigungen gilt die verschuldensunabhängige Haftung des Arbeitgebers. Ist die belästigende Handlung eines Vorgesetzten z. B. eine sexuelle Forderung, die mit beruflichen Vor- und Nachteilen verknüpft ist, und führt diese zu einer signifikanten negativen Änderung der Arbeitsbedingungen

nachweisen kann, dass bei ihm kein Verschulden vorliegt. Zivilgesetzbuch der Volksrepublik China, ZChinR 2020, S. 207, 399.

16 Vgl. CAO Yanchun (曹艳春), Eine ökonomische Analyse der Haftung des Arbeitgebers bei sexueller Belästigung am Arbeitsplatz (职场性骚扰雇主责任的法经济 学分析)，Contemporary Law Review (当代法学)，2008, Nr.9, S.95, 96 ff.; ZHANG Xinbao (张新宝)/GAO Yanzhu (高燕竹), Die Hauptfragen beim Gesetz gegen sexuelle Belästigung, : “性骚扰法律规制的主要问题” , The Jurist (法学 家), 2006, Nr. 4, S. 65,72; ZHOU Yingjiang (周应江), Anwendung der Arbeitgeber's Haftung auf sexuelle Belästigung am Arbeitsplatz (论雇主责任在职场性骚 扰行为上的适用), Journal of Chinese Women's Studie (妇女研究论丛), 2010, Nr. 5, S. 20, 25.; LU Jiefeng (Fn. 6), S. 92; WANG YiChun (王毅纯), Mögliche Regelungsmodelle für sexuelle Belästigung im Buch des Persönlichkeitsrechts des ZGB (民法典人格权编对性骚扰的规制路径和规则设计), Henan Social Sciences (河南社会科学), 2019, Nr. 7, S. 49, 55. 
für das Opfer - wie z. B. eine Entlassung oder eine gescheiterte Beförderung, haftet das Unternehmen automatisch für die Handlungen des Vorgesetzten. Dabei spielt es keine Rolle, ob der Arbeitgeber überhaupt von den Vorfällen Kenntnis hatte oder hätte haben müssen. ${ }^{17}$ Unerheblich hierfür ist zudem, ob der Arbeitgeber Maßnahmen gegen sexuelle Belästigung ergriffen hat. ${ }^{18}$ Für „Hostile environment harassment“"19 gilt das Prinzip der verschuldensabhängigen Haftung. Der Arbeitgeber haftet nur, wenn er von den Belästigungsvorfällen Kenntnis hatte oder hätte haben müssen und trotzdem keine angemessenen Maßnahmen dagegen ergriffen hat. ${ }^{20}$ $\mathrm{Zu}$ beachten ist, dass nicht jede von einem Vorgesetzten begangene sexuelle Belästigung als "Quid pro quo harassment" klassifiziert wird. Führt die in Rede stehende Belästigung zu keiner negativen Änderung der Arbeitsbedingungen, wird eine solche Belästigung als „Hostile environment harassment" definiert.

Obwohl die Voraussetzungen für die zwei oben genannten Haftungen unterschiedlich sind, haben die betroffenen Arbeitnehmer/innen den Anspruch auf Ausgleich aller durch die sexuelle Belästigung entstandenen Vermögensschäden und immateriellen Schäden. In der Tat sind diejenigen, die sich für die Übertragung US-amerikanischer Regelungen ins chinesische Recht einsetzen, eher an der Rechtsfolge für den Arbeitgeber interessiert als an den Tatbeständen bzw. dem Anwendungsbereich von den entsprechenden Haftungen. Denn die verschuldensunabhängige Haftung stelle einen Anreiz für den Arbeitgeber dar, nur geeignete und zuverlässige Personen in eine Position als Vorgesetzten einzustellen und die angemessenen Maßnahmen rechtzeitig zu ergreifen ${ }^{21}$. Aufgrund dessen schlugen sie

17 Henson v. City of Dundee, 682 F. 2d 897, 909 (11 ${ }^{\text {th }}$ Cir. 1982); Miller v. Bank of America, 600 F.2d 211, 213 (9 th $^{\text {th }}$ Cir. 1979); Sparks b. Regional Medical Center Board, 792 F. Supp. 735, 743 (N.D. Ala. 1992).

18 Kotcher v. Rosa and Sullivan Applicance Center Inc., 957 F. 2d 59, 62 (2nd Cir. 1992).

19 Meritor Savings Bank, FSB v. Vinson, 477 U.S. 57, Supreme Court of the United States (June 19th, 1986).

20 Henson v. City of Dundee, 682 F. 2d 897, 910 (11 $1^{\text {th }}$ Cir. 1982); Rabidue v. Osceola Refining Co., 805 F. 2d 611, 621 (6 ${ }^{\text {th }}$ Cir. 1986); Katz v. Dole, 709 F.2d 251, 256 (4 $4^{\text {th }}$ Cir. 1983).

21 CAO Yanchun (Fn. 16), S. 97; ZHANG Xinbao/GAO Yanzhu (Fn. 16); LU Jiefeng (卢杰峰), Die Analyse der US-amerikanischen Rechtsprechung für die Haftung des Arbeitgebers bei sexueller Belästigung am Arbeitsplatz (美国职场性骚扰雇主 责任的判例法分析), Journal of Chinese Women's Studies (妇女研究论丛), 2016, Nr. 2, S. 71, 77 ff. 
vor, auf $₫ 1191$ S. 1 ZGB (vgl. $\$ 34$ S. 1 HaftpflichtG) ${ }^{22}$ als unterstützende Rechtsgrundlage die Haftung für "Quid pro quo harassment" und $\$ 1198$ ZGB (vgl. $\$ 37$ HaftpflichtG) ${ }^{23}$ als unterstützende Rechtsgrundlage die Haftung für „Hostile environment harassment“ zu begründen.

\section{Keine Notwendigkeit der Übertragung der Haftungsregel für „Quid pro quo harassment"}

Die Übertragung der Haftungsregel für "Quid pro quo harassment“ wird aber kritisch gesehen, wenn man auf die Theorie der Rechtsvergleichung zurückgreift. Demnach sind alle Länder mit ähnlichen zu bewältigenden Problemen im Zusammenleben konfrontiert, deren Lösungen nicht unbedingt in gleichen Rechtsinstituten zu finden sind. ${ }^{24}$ Wenn eine konkrete Regelung aus dem US-amerikanischen Recht in China fehlt, heißt das nicht unbedingt, dass diese Regelung ins chinesische Recht umgesetzt werden muss. Eine solche Umsetzung wird erst dann notwendig, wenn die gleichen oder ähnlichen Probleme entweder nicht durch andere Rechtsinstitute oder durch andere Mechanismen gelöst werden können. ${ }^{25}$ In diesem Zusammenhang muss zunächst geklärt werden, was für ein Problem durch den Begriff „Quid pro quo harassment“ und die damit verbundene

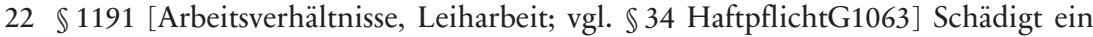
Mitarbeiter einer Arbeitgebereinheit einen anderen durch die Ausführung der Arbeitsaufgaben, haftet die Arbeitgebereinheit für die Verletzung von Rechten. Nachdem die Arbeitgebereinheit für die Verletzung. Zivilgesetzbuch der Volksrepublik China, ZChinR 2020, S. 207, 402.

$23 \$ 1198$ [Sorgfaltspflichten auf Betriebsplätzen oder öffentlichen Plätzen;] Ist der Betreiber [oder] der Verwalter der Betriebsplätze [oder] öffentlicher Plätze wie etwa Hotels, Kaufhäuser, Banken, Bahnstationen, Flughäfen, Sportstadien [und] Vergnügungsstätten oder der Organisator von Massenaktivitäten der Pflicht zur Gewährleistung der Sicherheit nicht vollständig nachgekommen, sodass andere geschädigt werden, haftet er für die Verletzung von Rechten. Wird ein anderer durch die Handlung eines Dritten geschädigt, haftet der Dritte für die Verletzung von Rechten; ist der Betreiber, der Verwalter oder der Organisator der Pflicht zur Gewährleistung der Sicherheit nicht vollständig nachgekommen, haftet er entsprechend ergänzend. Nachdem der Betreiber, der Verwalter oder der Organisator ergänzend gehaftet hat, kann er vom Dritten Ausgleich verlangen. Zivilgesetzbuch der Volksrepublik China, ZChinR 2020, S. 207, 404.

24 Konrad Zweigert / Hein Kötz,, Einführung in die Rechtsvergleichung, Thübingen 1996, S. 33.

25 Konrad Zweigert / Hein Kötz (Fn. 24), S. 34 ff. 
verschuldensunabhängige Haftung im US-amerikanischen Rechtsrahmen bewältigt wird.

Anders als das deutsche oder das chinesische Arbeitsvertragsrecht ist das US-amerikanische Arbeitsrecht von dem Grundsatz „employment at will“ geprägt. Dies bedeutet: Grundsätzlich kann sowohl der Arbeitgeber als auch der Arbeitnehmer jederzeit das Arbeitsverhältnis mit oder ohne Grund beenden. ${ }^{26}$ Somit könnte eine Mitarbeiterin durch das Arbeitsvertragsrecht auch dann nicht vor der Kündigung oder Versetzung geschützt werden, wenn die Kündigung eng mit einer sexuellen Belästigung verbunden ist. Unzulässig ist aber eine Kündigung aufgrund der Rasse, des Geschlechts oder der Religion etc. Eine solche Kündigung stellt eine Diskriminierung dar. Um dieses Problem zu bewältigen, strebten feministische Juristen in den USA (insbesondere Catharine A. MacKinnon) an, eine Kündigung oder Versetzung, die im Zusammenhang mit sexueller Belästigung steht, als Diskriminierung im Sinne von Titel VII des Civil Rights Act zu interpretieren. ${ }^{27}$ Demnach stellt eine Diskriminierung eine unerlaubte Handlung im Rahmen des Deliktsrechts dar. In diesem Zusammenhang kommt „relationship - based strict liability“ erst in Betracht.

Im Vergleich zu dem Arbeitsrecht der USA verlangt das chinesische Arbeitsvertragsgesetz für jede Kündigung seitens des Arbeitgebers das Vorliegen eines gesetzlichen Kündigungsgrunds. Mögliche Gründe sind z. B. die fehlende Fähigkeit zur Erfüllung der Arbeitspflicht, ein erheblicher Verstoß gegen betriebsinterne Regelungen, eine erhebliche Vernachlässigung der Aufgaben, welche den Interessen des Arbeitgebers substantiell geschadet hat. Kündigt der Arbeitgeber das Arbeitsverhältnis wegen der Ablehnung einer sexuellen Belästigung, ist eine solche Kündigung unwirksam. Somit hat der betroffene Arbeitnehmer ein Wahlrecht zwischen der Klage auf Wiedereinstellung oder auf die verdoppelte gesetzliche Abfindung ( $\left(87 \mathrm{ArbVG}^{28}\right)$. Ebenfalls eingeschränkt wird das Versetzungsrecht des Arbeitgebers. Gemäß $₫ 35$ ArbVG $^{29}$ ist eine Änderung des Arbeitsvertrags nur

26 Peggie R. Smith, Ann C. Hodges, Susan J. Stabile, Rafael Gely, Principle of Employment Law, West, 2009, S. 23.

27 Catharine Mackinnon (Fn. 12), S. 215.

$28 \$ 87$ ArbVG, Wenn der Arbeitgeber den Arbeitsvertrag entgegen diesem Gesetz kündigt oder beendet, muss er dem Arbeitenden eine Entschädigung in Höhe des Doppelten der wirtschaftlichen Abfindung nach $\$ 47$ zahlen, ZChinR, 2008, 2.

$29 \$ 35$ ArbVG, Arbeitgeber und Arbeitender können eine Änderung des vereinbarten Inhalts des Arbeitsvertrags aushandeln. Die Änderung eines Arbeitsvertrags bedarf der Schriftform. Arbeitgeber und Arbeitender behalten je ein Exemplar der Arbeitsvertragsurkunde nach der Änderung. ZChinR, 2008, 2. 
zulässig, wenn eine Vereinbarung durch beide Vertragsparteien getroffen wird. Eine Versetzungsklausel, wonach der Arbeitgeber das Gesamtbild der vereinbarten Tätigkeit einseitig ändern kann, ist in der Rechtspraxis anerkannt, deren Ausübung unterliegt aber einer Billigkeitskontrolle. So schreibt die Beantwortung einiger Fragen über arbeitsrechtliche Streitigkeiten (Höheres Volksgericht Shanghai) ${ }^{30}$ vor, dass eine einseitige Versetzung aus sachlichen Gründen erfolgen soll. Zudem sollen die Arbeitsbedingungen, einschließlich der Arbeitsvergütung, nicht durch die Versetzung verschlechtert werden. Schließlich ist es verboten, eine Versetzung als ein vergeltendes oder beleidigendes Mittel zu nutzen. Kündigt der Arbeitnehmer wegen einer vergeltenden oder beleidigenden Versetzung einseitig das Arbeitsverhältnis, hat er einen Anspruch auf die verdoppelte gesetzliche Abfindung ( $\$ 87$ ArbVG), was als Sanktion gegen den Arbeitgeber dient. Für "Quid pro quo harassment" lässt sich daraus ableiten, dass eine vergeltende und beleidigende Versetzung gerade vorliegt, wenn die in Rede stehende Versetzung an eine sexuelle Belästigung anknüpft.

Ein weiterer Grund für die Ablehnung der Übertragung liegt darin, dass $\$ 1191$ ZGB kaum geeignet ist, die Haftung des Arbeitgebers zu begründen. Demnach kommt eine Haftung des Arbeitgebers in Betracht, wenn sein Arbeitnehmer in Ausführung der Verrichtung eine tatbestandsmäßige unerlaubte Handlung begangen hat. Anders als bei $\$ 831$ Abs. 1 BGB kann sich der Arbeitgeber nicht von der Haftung befreien, auch dann nicht, wenn er bei der Auswahl und Überwachung der Hilfsperson die erforderliche Sorgfalt hat walten lassen. Die Anwendung des $\$ 1191$ ZGB setzt also

30 Höheres Volksgericht Shanghai: Beantwortung der einigen Fragen über arbeitsrechtliche Streitigkeiten (上海市高级人民法院关于审理劳动争议案件若干问题 的解答) , Hugaofa Min 1 (2006), Nr. 17(沪高法民一[2006]17 号). Dem folgt das Höhere Volksgericht Jiangsu (江苏省高级人民法院劳动争议案件审理指南, v. 17.5.2010), Höheres Volksgericht Guangdong und Schiedskommission für Arbeitsstreitigkeiten Guangdong; Gesprächsnotizen über einige Fragen der arbeitsrechtlichen Streitigkeiten (广东省高级人民法院、广东省劳动人事争议仲裁委员 会关于审理劳动人事争议案件若干问题的座谈会纪要，Yuegaofa [2012] Nr. 284,), Höheres Volksgericht Sichuan: Mittelung über Drucksache der Beantwortung über einige Fragen der arbeitsrechtlichen Streitigkeiten, (四川省高级人 民法院民事审判第一庭关于印发《关于审理劳动争议案件若干疑难问题的解 答》的通知 , Chuangaofa Min 1 (2016), Nr.1), Höheres Volksgericht Beijing und Schiedskommission für Arbeitsstreitigkeiten Beijing: Beantwortung einiger Fragen über arbeitsrechtliche Streitigkeiten (北京市高级人民法院、北京市劳动 争议仲裁委员会关于审理劳动争议案件法律适用问题的解答，v. 24.04.2017) und Chongqing: Gesprächsnotizen der Rechtsanwendung im Bereich der arbeitsrechtlichen Streitigkeiten (重庆市六部门劳动争议案件法律适用问题专题座谈会 纪要 (二) , v. 11.9.2017). 
zunächst voraus, dass nicht der Arbeitgeber selbst, sondern sein Mitarbeiter (Gehilfe) die unerlaubte Handlung begangen hat. Für „Quid pro quo harassment“ besteht die unerlaubte Handlung aber in der Kündigung oder Versetzung. Nach dem Verständnis des chinesischen Arbeitsrechts können Kündigung oder Versetzung, welche als Beendigung oder Änderung des Arbeitsverhältnisses verstanden werden, nur durch die Vertragsparteien erfolgen. ${ }^{31}$ Daraus lässt sich ableiten, dass der Schädiger bei „Quid pro quo harassment" der Arbeitgeber selbst ist. In diesem Fall findet $\$ 1191$ ZGB keine Anwendung. ${ }^{32}$ Zusammenfassend ist zu sagen, dass der Schutz vor den meisten ungerechtfertigten beruflichen Nachteilen schon durch das chinesische Arbeitsvertragsrecht gewährleistet wird. Die Eröffnung der Haftungsregeln im Deliktsrecht ist deshalb unnötig.

3. Für „Hostile environment harassment": $\$ 1191$ und $\$ 1198$ ZGB (vgl. SS 34, 37 HaftpflichtG)

In vielen repräsentativen Fällen, in denen der Arbeitnehmer vom Arbeitgeber Schadenersatz verlangt, wird einer solcher Anspruch des Arbeitnehmers verneint. Begründet wird dies damit, dass der Arbeitgeber die unerlaubte Handlung (sexuelle Belästigung) nicht begangen hat. ${ }^{33}$ Dies ist unberechtigt. In Bezug auf „Hostile environment harassment" wird der Schaden des betroffenen Arbeitnehmers nicht lediglich durch die unwillkommenen sexuellen Handlungen selbst herbeigeführt. Vielmehr spielt die Unterlassung des Arbeitgebers eine elementare Rolle. Dies liegt insbesondere dann vor, wenn der Arbeitgeber das in Frage stehende Verhalten nicht als sexuelle Belästigung einstuft und nichts unternimmt. Damit wird dem Täter ein Anreiz geschaffen, wiederholte und erhebliche sexuelle Handlungen zu begehen. In dieser Situation ist die Kausalität zwischen dem Unterlassen des Arbeitgebers und dem Schaden des Arbeitnehmers

31 LIU Jun (刘俊), Arbeits- und Sozialrecht (劳动与社会保障法), Beijing 2018, S. 100.

32 Ähnlicher Ansicht WANG Xianyong (王显勇), Rechtsinstitute gegen sexuelle Belästigung am Arbeitsplatz in der Zeit des ZGB (民法典时代工作场所性骚扰的法 律规制), Law of Science (法学), 2020, Nr. 1, S. 133, 140; XIA Limin (夏利民 ) / GUO Hui ( 郭辉), Kritik gegen die Anwendung der Haftung für den Verichtungsgehilfen auf sexuelle Belästigung am Arbeitsplatz(职场性骚扰雇主替代责任 说质疑), Hebei Faxue (河北法学), März, 2012, S. 29, 34.

33 Urteil des Volksgerichts Chengdu Wuhou im Rechtsstreit über die sexuelle Belästigung Xu gegen Liu, (2019), Chuan 0107 Min Chu Nr. 1407. 
unzweifelhaft. In diesem Zusammenhang verdient der Vorschlag der oben genannten Literaturansicht ${ }^{34}$ Zustimmung. Demnach haftet der Arbeitgeber bei „Hostile environment harassment“ verschuldensabhängig. Das Verschulden des Arbeitgebers liegt also nicht vor, wenn er mit vernünftiger Sorgfalt alle Maßnahmen ergriffen hat, um sexuell belästigendes Verhalten $\mathrm{zu}$ verhindern und zu ahnden oder sich der betroffene Arbeitnehmer unvernünftigerweise dieser Mechanismen nicht bedient hat. Einerseits wird der Arbeitgeber dafür belohnt, alle entsprechenden Maßnahmen zu ergreifen. Anderseits muss sich der betroffene Arbeitnehmer auch um eine Schadensminderung kümmern. ${ }^{35}$

Nun ist es fraglich, welche gesetzlichen Vorschriften eine solche Haftung stützen könnten. Im Bereich des Deliktsrechts denkt man zunächst an $\$ 1191$ ZGB. Eine Haftung des Arbeitgebers nach dieser Vorschrift kommt in Betracht, wenn ein Mitarbeiter einen anderen bei der Ausführung von Arbeitsaufgaben schädigt. Vor dem Erlass des ZGB orientierte sich die Feststellung, ob ein Schaden „durch Ausführung der Arbeitsaufgaben“ vorlag, an einem objektiven Maßstab. ${ }^{36}$ Gemäß $\$ 9$ Abs. 2 S. 1 Auslegung zu Entschädigung des persönlichen Schadens (a. F.) ${ }^{37}$ wurde eine schädigende Handlung dem Arbeitgeber zugerechnet, soweit die Handlung im Rahmen der Anweisungen oder mit Genehmigung des Arbeitgebers begangen wurde. Unter „durch Ausführung der Arbeitsaufgaben“ gehörten auch die Handlungen, die zwar außerhalb des Bereichs der Anweisungen oder der Genehmigung lagen, aber mit den ausführenden Arbeitsaufgaben in einem inneren Zusammenhang standen oder den Anschein erweckten, dass der Schädiger sein Arbeitsaufgebe ausführte ( $\$ 9$ Abs. 2 S. 2, Auslegung zu Entschädigung des persönlichen Schadens). Diese Vorschrift wurde im Dezember 2020 vom Obersten Volksgericht gestrichen. ${ }^{38}$

34 Vgl. LU Jiefeng (Fn. 6), S. 92 ff.; CAO Yanchun (Fn. 16), S. 95.; WANG YiChun (Fn. 16), S. 54.

35 Faragher v. City of Boca Raton, 118 S. Ct. 2275, 2293; Burlington Industries, Inc. V. Ellerth, 118 S. Ct. 2257, 2270.

36 WANG Liming (王利明), Forschung zum Deliktshaftungsgesetz (Band II) (侵权 责任法研究(下卷)), Beijing 2011, S.99. CHENG Xiao (程啸), Deliktshaftungsrecht (侵权责任法), Beijing 2021, S. 467.

37 Auslegung des OVG zu einigen Fragen für die Fälle über persönliche Schäden, erlassen am 4. 12. 2003 (最高人民法院关于审理人身损害赔偿案件适用法律若干 问题的解释).

38 OVG: Beschluss über die Änderung der justiziellen Auslegungen im Bereich des Zivilrechts, Fashi (2020) Nr. 17. (最高人民法院关于修改《最高人民法院关于在 民事审判工作中适用〈中华人民共和国工会法〉若干问题的解释》等二十七件 民事类司法解释的决定). 
Nach Auslegung des ständigen Ausschusses des Nationalen Volkskongresses ist $\mathbb{1 1 9 1}$ ZGB anwendbar, wenn die schädigende Handlung im Rahmen der Anweisungen oder Genehmigungen liegt. ${ }^{39}$

Für die Fälle sexueller Belästigung am Arbeitsplatz lässt sich daraus folgendes ableiten: Ordnet der Arbeitgeber einem Vorgesetzten an, einen großen Auftrag um jeden Preis zu gewinnen, ohne moralische Regeln zu berücksichtigen und nötigt der Vorgesetzte dann eine Arbeitnehmerin dazu, bei den Verhandlungen mit dem Geschäftspartner zu schlafen, ist die belästigende Handlung des Vorgesetzten dem Arbeitgeber zurechnen. Das Gleiche gilt, wenn der Arbeitgeber den betroffenen Arbeitnehmer die Belästigung ertragen lassen hat, obwohl der betroffene Arbeitnehmer diese meldete. Schwierigkeiten ergeben sich jedoch, wenn die Belästigenden Handlungen nicht vom Arbeitgeber angewiesen oder genehmigt werden. CHENG Xiao ist der Auffassung, dass die Zurechnung eines schädigendes Verhaltens voraussetzt, dass zwischen dem Verhalten und die Durchführung der Arbeitsaufgaben ein innerer Zusammenhang steht. ${ }^{40}$ Maßgeblich für die Beurteilung des inneren Zusammenhangs sei vor allem, ob die schädigenden Handlung ein Risiko darstelle, welches hauptsächlich durch Ausführung einer Arbeitsaufgabe entstehe oder nur ein allgemeines Risiko darstelle. ${ }^{41}$ Meines Erachtens könnte sich dieses Kriterium auf die Fälle sexueller Belästigung übertragen lassen. Wenn der Arbeitgeber eine Person in seinem Unternehmen als Vorgesetzten einstellt, verschafft er dieser Person auch die Möglichkeit, Direktionsrechte auszuüben. Die Untergebenen sind verpflichtet, den Anordnungen des Vorgesetzten über die Einzelheiten der Arbeitsleistung Folge zu leisten. Zum einen ermöglicht ein solch hierarchisches Verhältnis dem Vorgesetzten, mit seinen Untergebenen unter vier Augen zu sprechen, was das Risiko eines unerwünschten körperlichen Berührens oder einer unerwünschten sexuellen Aufforderung erhöht. Zum anderen fällt es einem Untergeordneten schwer, die mögliche bevorstehende sexuelle Belästigung abzuwenden. Eine Arbeitnehmerin kann normalerweise nicht eine angeordnete Dienstreise ablehnen, auch wenn sie weißt, dass sie während der Dienstreise von ihrem Vorgesetzten sexuell belästigt werden könnte. Angesichts dessen sollten die belästigenden

39 HUANG Wei (Hrsg.) (黄薇), Auslegung zum Buch: Haftung für die Verletzung von Rechten, ZGB (中华人民共和国民法典侵权责任编释义), Beijing 2020, S. 85.

40 CHENG Xiao (Fn. 36), S. 467.

41 CHENG Xiao (Fn. 36), S. 467; Ähnlicher Ansicht CAO Yanchun (曹艳春), Der Umfang der Ausführung der Arbeitsaufgaben in der Haftung für den Verrichtungsgehilfen (雇主替代责任的雇佣范围之考量), Law Science Magazine (法学杂 志), 2008. Nr. 4, S. 30, 62 . 
Handlungen, die von einem Vorgesetzten durch Macht begangen werden, dem Arbeitgeber zugerechnet werden. Dagegen stehen die Belästigungen durch gleichrangige Kollegen nicht im Zusammenhang mit relevanten Maßnahmen, die nur ein Vorgesetzter durch Macht ergreifen kann. Ein Arbeitnehmer kann auch die möglichen bevorstehenden Belästigungen verhindern. Folglich sollten belästigende Handlungen durch gleichrangige Kollegen nicht dem Arbeitgeber zugerechnet werden.

Somit greift $\$ 1191$ ZGB dann ein, wenn ein Vorgesetzter seinen Untergebenen sexuell belästigt. Der Arbeitgeber muss den durch sexuelle Belästigung entstandenen Schaden ersetzen, auch wenn der Arbeitgeber mit vernünftiger Sorgfalt alle zumutbaren Maßnahmen ergriffen hat. Eine solche Rechtsfolge könnte vielleicht nicht geeignet sind, das oben genannte Problem zu lösen. In der Literatur wird die Ansicht vertreten, dass sich der Arbeitgeber die Motivation verlieren würde, entsprechende Maßnahmen zu ergreifen, wenn man ihm die Möglichkeit verwehrt, sich von der Haftung zu befreien. ${ }^{42}$ Dies ist jedoch ein Trugschluss, denn gerade eine strengere Haftung dazu führen würde, dass der Arbeitgeber versuchen wird, die Entstehung der Schadensfälle möglichst zu vermeiden.

Stellt man zunächst die Anwendung von $\mathbb{1} 1191$ ZGB dahin, wäre zu prüfen, welche Vorschriften noch anwendbar sein könnten, wenn eine sexuelle Belästigung im Sinne von „Hostile Environment harassment“ durch einen gleichrangigen Kollegen begangen wird. Im US-amerikanischen Recht haftet der Arbeitgeber bei einer solchen Belästigung für alle Schadensfolgen. Sofern diese Rechtsfolge im chinesischen Rechtsrahmen verwirklicht werden soll, kommen zunächst $\$ \mathbb{S} 1168^{43}$ und 1169 S. 1 ZGB ${ }^{44}$ als mögliche Rechtsgrundlage in Betracht, wonach alle, die an der unerlaubten Handlung beteiligt waren, als Gesamtschuldner für die Schadensfolge haften sollen. Gemäß $\$ \$ 1168,1169$ ZGB kann der Arbeitnehmer vom Arbeitgeber Schadenersatz verlangen, soweit der Arbeitgeber als Mittäter $(\$ 1168 \mathrm{ZGB})$, Anstifter oder Gehilfe $(\$ 1169 \mathrm{ZGB})$ an der unerlaubten Handlung mitgewirkt hat. Dies verlangt aber eine Kommunikation

42 CAO Yanchun (Fn. 16), S. 97.

$43 \$ 1168$ [Gemeinschaftliches Delikt] Haben mehrere Personen gemeinsam eine rechtsverletzende Handlung vorgenommen [und] dadurch einen anderen geschädigt, haften sie als Gesamtschuldner. Zivilgesetzbuch der Volksrepublik China, ZChinR 2020, S. 207, 399.

$44 \$ 1169$ [Anstiftung und Beihilfe; vgl. $\$ 9$ HaftpflichtG] Wer einen anderen zur Vornahme einer rechtsverletzenden Handlung anstiftet [oder] ihm dabei hilft, haftet mit dem Handelnden als Gesamtschuldner. Zivilgesetzbuch der Volksrepublik China, ZChinR 2020, S. 207, 399. 
zwischen Täter und dem Arbeitgeber ${ }^{45}$, an der es bei einer sexuellen Belästigung durch Dritte normalerweise fehlt. Denn der Arbeitgeber hat normalerweise keine Absicht, mit dem Täter den betroffenen Arbeitnehmer zusammen sexuell zu belästigen. Daraus kann man schließen, dass das chinesische Deliktsrecht keine gesetzliche Regelung als Rechtsgrundlage bietet, wonach der Arbeitgeber für alle Schadensfolgen haften soll.

Denkbar wäre auch eine Teilschuldnerschaft. Würde der Arbeitgeber wegen Verletzung der Pflichten i. S. v. $\$ 1010$ ZGB einen Teil der durch die Belästigung verursachten Schäden ersetzen, würde auch für ihn ein Anreiz geschaffen, angemessene Maßnahmen gegen sexuelle Belästigung zu ergreifen. Als mögliche Rechtsgrundlage könnte man an $\$ 1198$ ZGB denken (Ergänzende Haftung wegen Verletzung der Verkehrspflicht). Diese Vorschrift regelt den Ersatz des durch den Dritten verursachten Schadens in Fällen, in denen die Verkehrssicherungspflichten nicht vollständig erfüllt werden. Zu den Personen, die gemäß $\$ 1198$ ZGB Verkehrssicherungspflichten treffen, gehören die Betreiber und Verwalter von Betriebsplätzen oder öffentlichen Plätzen, wie etwa Hotels, Kaufhäuser, Banken, Bahnstationen, Flughäfen und Sportsstadien oder Organisatoren von Massenaktivitäten. Fraglich ist es jedoch, ob ein Unternehmen gemäß $\$ 1198$ ZGB verpflichtet ist, die Rechtsgüter seiner Beschäftigten zu schützten. Eine solche Pflicht wurde im Fall „Li vs. General Fushi“46, der als repräsentativer Fall im Amtsblatt des OVG veröffentlich wurde, bejaht. Begründet wurde dies damit, dass die Beklagte, die die Arbeitsmittel stellte und die Praktikanten bei der Arbeit anwies, auch den Leib und die Gesundheit der Praktikanten schützen solle. Somit wurde der Anwendungsbereich des $\$ 1198$ ZGB erweitert. ${ }^{47}$ In diesem Sinn ist der Arbeitgeber gemäß $\$ 1198$ ZGB verpflichtet, seine Beschäftigten, einschließlich seiner Arbeitnehmer und Arbeitnehmerinnen, vor sexueller Belästigung zu schützen. Hat der Arbeitgeber bei sexueller Belästigung keine Maßnahme i. S. v. $\$ 1010$ S. 2 ZGB ergriffen, stellt dies die Verletzung einer Verkehrssicherungspflicht i. S. v. $\$ 1198$ ZGB dar. In dieser Situation haftet der Täter als Hauptersatzpflichtiger und der Arbeitgeber als Nebenersatzpflichtiger.

45 CHENG Xiao (Fn. 36), S. 385.

46 Urteil des Zweiten Mittleren Volksgerichts Shanghai im Rechtsstreit um die Verkehrssicherungspflicht Li Shuaishuai gegen Shanghai General Fushi Co., (2015), Hu Er Min Yi Min 0107 Min Zhong Nr. 187/Amtsblatt des OVG, 2015, Nr. 12.

47 Eine solche Erweiterung wird auch in der Literatur anerkannt. Vgl. CHENG Xiao (Fn. 36), S. 520. 


\section{Vertraglicher Anspruch bei sexueller Belästigung am Arbeitsplatz}

\section{Verstoß gegen $\$ 1010$ S. 2 ZGB als positive Vertragsverletzung}

Liegt eine Verletzung einer Vertragspflicht vor, wenn der Arbeitgeber keine Maßnahmen ergriffen hat, obwohl er von einer sexuellen Belästigung erfahren hat? Zur Beantwortung dieser Frage muss zunächst geklärt werden, welche Pflichten der Arbeitgeber bei der Erfüllung des Arbeitsvertrags hat. Wie im deutschen Recht besteht die Hauptpflicht des Arbeitgebers darin, dem Arbeitnehmer eine Vergütung für seine Arbeitsleistung zu zahlen ( $\$ 30$ Abs. 1 ArbVG ${ }^{48}$ ). Eine Regelung, die Schutzpflichten im Schuldverhältnis bzw. Arbeitsverhältnis ausdrücklich vorsieht, wie etwa $\$ 241$ Abs. 2 und $\$ 618$ BGB, existiert im chinesischen Zivilrecht noch nicht. Allgemein anerkannt ist aber, dass der Arbeitgeber auf den Leib, die Gesundheit und die Würde des Arbeitnehmers Rücksicht nehmen muss. ${ }^{49}$ Meines Erachtens könnte sich eine solche Schutzpflicht auch aus $\$ 509$ $Z_{\mathrm{GB}^{50}}$ ergeben. Die darin normierte Nebenpflicht, die die Vertragsparteien bei der Vertragserfüllung nach dem Grundsatz von Treu und Glauben erfüllen müssen, sollte auch die Rücksichtnahme der Rechtsgüter und Interessen der jeweils anderen Parteien umfassen. ${ }^{51}$ Da eine sexuelle Belästi-

$48 \$ 30$ ArbVG, Der Arbeitgeber muss gemäß dem im Arbeitsvertrag Vereinbarten und gemäß den staatlichen Vorschriften den Arbeitenden unverzüglich das volle Arbeitsentgelt zahlen. ZChinR 2008, 2.

49 YUAN Zhonghua (袁中华), Anspruchsgrundlage im Arbeitsrecht (劳动法上请求 权体系之建构), Global Law Review (环球法律评论), 2020, Nr. 6, S. 88, 94.

$50 \$ 509$ [Vertragspflichten und Nebenpflichten] Die Parteien müssen nach der Vereinbarung ihre Pflichten in vollem Umfang erfüllen Die Parteien müssen unter Wahrung des Grundsatzes von Treu und Glauben aufgrund der Natur [und] des Zwecks des Vertrags und der geschäftlichen Gebräuche die Pflichten zu Mitteilungen, zur [gegenseitigen] Unterstützung, zur Geheimhaltung und anderem erfüllen. Die Parteien müssen im Verlauf der Vertragserfüllung vermeiden, Ressourcen zu verschwenden, die Umwelt zu verschmutzen und die Ökologie zu zerstören. Zivilgesetzbuch der Volksrepublik China, ZChinR 2020, S. 207, 290.

51 Vgl. CUI Jianyuan (崔建远), Allgemeinteil des Vertragsrechts (Band I), (合同法总 论 (上卷）), Beijing 2011, S. 239; HAN Shiyuan (韩世远), Allgemeinteil des Vertragsrechts (合同法总论)，Beijing 2011, S. 250; YIN Zhiqiang (尹志强), Die Legitimität und der Anwendungsbereich des immateriellen Schadenersatzes wegen Pflichtverletzung (论违约精神损害赔偿的正当性及适用范围), Journal of CUPL (中国政法大学学报), 2014, Nr. 6, S. 109, 115; CHI Ying (迟颖), Forschung über Nebenpflichten im chinesischen Vertragsgesetz (我国合同法上附随义务之正本 清源 一以德国法上的保护义务为参照), Political Science and Law (政治与法律), 2011, Nr. 7, S. 128, 129. 
gung das Persönlichkeitsrecht des Arbeitnehmers verletzt, ist der Arbeitgeber gemäß $\$ 509$ ZGB verpflichtet, den Arbeitnehmer vor sexuellen Belästigungen zu schützen. In diesem Zusammenhang stellt $\$ 1010$ S. 2 ZGB eine gesetzlich konkretisierte Ausprägung der Schutzpflicht hinsichtlich des Persönlichkeitsrechts des Arbeitnehmers dar. Daher führt ein Verstoß gegen $\$ 1010$ S. 2 ZGB zu einer Haftung wegen positiver Vertragsverletzung. Eine positive Vertragsverletzung liegt selbstverständlich auch dann vor, wenn der Arbeitnehmer durch den Arbeitgeber selbst belästigt wird.

\section{Anspruch auf Schadenersatz wegen Vertragsverletzung}

Grundsätzlich sind die Rechtsfolgen von Vertragsverletzungen in $\$ 577$ ZGB $^{52}$ geregelt, wonach die Nichterfüllung der Schutzpflicht zum Schadensersatzanspruch führen kann. Bei sexueller Belästigung könnte der belästigte Arbeitnehmer Schadensersatz verlangen, sofern er sowohl (a) die Pflichtverletzung (d.h., dass der Arbeitgeber es versäumt hat, angemessene Maßnahmen zur Unterbindung der Belästigung zu ergreifen) als auch (b) die Kausalität zwischen den Schäden und der Pflichtverletzung nachgewiesen hat. Anders als im deutschen Arbeitsrecht braucht der Gläubiger nicht nachzuweisen, dass der Schuldner die Pflichtverletzung zu vertreten hat.

Unklar bleibt aber, ob der belästigte Arbeitnehmer einen Ersatz für den immateriellen Schaden gemäß $\$ 577$ ZGB verlangen kann, welcher durch die sexuelle Belästigung entstanden ist, wie z. B. Leiden, Schmerzen, Demütigung etc. Aus dem Wortlaut des $₫ 577$ ZGB lässt sich nicht ableiten, dass immaterielle Schäden vom Anwendungsbereich ausgeschlossen werden. Allerdings besteht bis jetzt keine Einigkeit darüber, ob der Schadenersatz i. S. v. $\$ 577$ ZGB überhaupt den immateriellen Schaden erfasst. ${ }^{53}$ Manche sind der Auffassung, dass die Abdeckung des immateriellen Scha-

$52 \$ 577$ [Formen der Haftung] Wenn eine Partei Vertragspflichten nicht erfüllt oder die Erfüllung der Vertragspflichten nicht den Vereinbarungen entspricht, haftet sie für die Vertragsverletzung [in Formen] wie etwa die Erfüllung fortzusetzen, Maßnahmen zur Abhilfe zu ergreifen oder den Schaden zu ersetzen. ZChinR 2020, S. 207, 303.

53 Ausführlich hierzu: Jin Zhao, Der immaterielle Schadensersatz wegen Pflichtverletzung, in: Yuanshi Bu, Der besondere Teil der chinesischen Zivilrechtskodifikation, S. $139 \mathrm{ff}$. 
dens $₫ 584 \mathrm{ZGB}^{54}$ nicht entspricht. ${ }^{55}$ Demnach müssen die durch eine Vertragsverletzung entstandenen Schäden für die Vertragsparteien vorhersehbar sein. Dieses Argument ist aber nur überzeugend, wenn es sich bei dem in Frage stehenden Vertrag um einen allgemeinen Vertrag, z. B. ein Kaufvertrag, handelt. Verzögert ein Verkäufer die Lieferung von Waren, kann der Käufer enttäuscht oder frustriert sein. Im Regelfall ist es aber ungewöhnlich, dass die Verzögerung zu Schmerz und Leiden des Käufers führt. Darum ist der immaterielle Schaden im Kaufvertrag normalerweise unabsehbar. Dies gilt aber nicht für die Verträge, deren Zweck in einem immateriellen Genuss liegt. Bei dem Abschluss solcher Verträge, wie etwa einem Reisevertrag, ist es dem Vertragspartner nicht unzumutbar, das Entstehen eines immateriellen Schadens durch eine Pflichtverletzung vorherzusehen. ${ }^{56}$ Gleiches gilt auch für Arbeitsverträge, welche von der persönlichen Abhängigkeit geprägt sind. ${ }^{57}$ Bei der Leistungserbringung steht fast jeder Arbeitnehmer während der Arbeitszeit in intensivem sozialen Kontakt mit Vorgesetzten und Kollegen. ${ }^{58}$ Studien zeigen, dass sexuelle Belästigung am Arbeitsplatz einen erheblichen negativen Einfluss sowohl auf den körperlichen als auch auf den psychischen Zustand des Opfers haben können. ${ }^{59}$ Dazu gehören Angst, Scham, Nervosität, Depressionen, Kopfschmerzen, Schlafstörungen, Gewichtsverlust oder -zunahme usw. Weitergehend führt

$54 \$ 584$ [Höhe des Schadensersatzanspruchs; vgl. $\$ 113$ VertragsG491] Erfüllt eine Partei Vertragspflichten nicht oder entspricht die Erfüllung nicht den Vereinbarungen, sodass die andere Seite geschädigt wird, muss der Betrag des Schadensersatzes dem durch die Vertragsverletzung herbeigeführten Schaden entsprechen, einschließlich des nach Vertragserfüllung zu erlangenden Gewinns; [der Betrag] darf aber den Schaden nicht übersteigen, den die vertragsverletzende Seite bei Abschluss des Vertrags als mögliche [Folge] einer Vertragsverletzung vorhergesehen hat oder vorhersehen musste. ZChinR 2020, S. 207, 304.

55 WANG Liming (王利明), Forschung über das Vertragsrecht (合同法研究), Beijing 2003, S. 670; ders., Forschung über den besonderen Teil des ZGB (民法分则 合同编立法研究), China Legal Science (中国法学), 2017, Nr. 2, S. 25, 44.

56 Vgl. CUI Jianyuan (崔建远), Der immaterielle Schadensersatz wegen Vertragsverletzung (论违约的精神损害赔偿), Henan Zhengfa Ganbu Guanli Xueyuan Xuebao (河南政法干部管理学院学报), 2018, Nr. 1, S. 48 ff; YIN Zhiqiang (Fn. 51), S. 117; HAN Shiyuan (韩世远), Immaterieller Schaden und Vertragshaftung (非 财产损害与合同责任), Science of Law (法学), 1998, Nr. 6, S. 27, 30.

57 Raimund Watermann, Arbeitsrecht, 19. Auflage, München 2018, S. 26 ff.

58 Tobias Mästle, Der zivilrechtliche Schutz vor sexueller Belästigung am Arbeitsplatz, Berlin 1999, S. 186.

59 TANG Can (唐灿), Sexuelle Belästigung am Arbeitsplatz: Ungleichheit in Machtausübung und Identitäten in zwanzig Fällen (工作场所中的性骚扰 : 多重权力和 身份关系的不平等), Journal of Chinese Women's Studie (妇女研究论丛), 2009, Nr. 11, S. 22, 29. 
die Duldung von Belästigung zu dem Verlust der Freude an der Arbeit. Daher ist es dem Arbeitgeber zumutbar, die durch die Duldung der sexuellen Belästigung verursachten Schmerzen und Leiden vorherzusehen. Somit kann der betroffene Arbeitnehmer Schmerzensgeld verlangen.

\section{Kündigung durch den betroffenen Arbeitnehmer}

$\$ 38$ Nr. 1 ArbVG sieht vor, dass der Arbeitnehmer den Arbeitsvertrag fristlos kündigen kann, wenn der Arbeitgeber die vereinbarten Arbeitsbedingungen nicht erfüllt. In dieser Situation hat der Arbeitnehmer einen Anspruch auf verdoppelte Abfindung ( $\$ 87$ ArbVG). Nach der Auslegung des Legislativkomitees des Ständigen Ausschusses des Nationalen Volkskongresses beziehen sich die Arbeitsbedingungen hauptsächlich auf die notwendigen Materialien und Technologien und andere Arbeitsbedingungen, welche dem Arbeitnehmer ermöglichen, die Arbeit zu erledigen. ${ }^{60}$ Unter die Arbeitsbedingungen i. S. v. $\$ 38$ Nr. 1 ArbVG $^{61}$ fällt auch die Arbeitsumgebung, wo das Persönlichkeitsrecht des Arbeitnehmers vor Verletzung geschützt werden soll. ${ }^{62}$ Daher sollte das Unterlassen von Maßnahmen zur Verhinderung sexueller Belästigung als „Nicht-Anbieten der Arbeitsbedingungen“i. S. v. $\$ 38$ Nr. 1 ArbVG angesehen werden. Somit könnte ein betroffener Arbeitnehmer oder eine betroffene Arbeitnehmerin eine verdoppelte Abfindung verlangen, wenn sie den Arbeitsvertrag aufgrund der sexuellen Belästigung kündigen.

60 XIN Chunying (信春鹰)/KAN Ke(阚珂), Auslegung des Arbeitsvertragsrechts der VR China (中华人民共和国劳动合同法释义), Beijing 2013, S. 56.

$61 \$ 38$ ArbVG, Wenn beim Arbeitgeber einer der folgenden Umstände vorliegt, kann der Arbeitende den Arbeitsvertrag kündigen $<14>$ : 1 . Wenn dem Arbeitenden nicht der vertragsgemäße Arbeitsschutz oder die vertragsgemäßen Arbeitsbedingungen gewährt werden, 2. wenn das Arbeitsentgelt nicht unverzüglich in vollem Umfang gezahlt wird, 3. wenn nicht nach dem Recht für den Arbeitenden Sozialversicherungsgebühren gezahlt werden, 4. wenn Regeln des Arbeitgebers, die gegen Gesetze oder sonstige Rechtsnormen verstoßen, [auch] die Rechtsinteressen des Arbeitenden verletzen, 5. wenn der Arbeitsvertrag nach $\$ 26$ Abs. 1 unwirksam ist, 6. wenn andere Umstände vorliegen, unter denen der Arbeitende nach Gesetzen oder sonstigen Rechtsnormen den Arbeitsvertrag kündigen kann. Wenn der Arbeitgeber den Arbeitenden mit Gewalt, Drohung oder rechtswidriger Freiheitsbeschränkung den Arbeitenden zur Arbeit zwingt oder mit regelwidrigen Anweisungen oder Befehlen zu gefährlicher Tätigkeit seine persönliche Sicherheit gefährdet, kann der Arbeitende sofort den Arbeitsvertrag kündigen, ohne den Arbeitgeber vorher zu unterrichten. ZChinaR, 2008, 2. 
Die Ausübung eines solchen Kündigungsrechts muss jedoch im Einklang mit dem Sinn und Zweck des $₫ 38$ ArbVG stehen. Dies kann sich aus der Analyse anderer Voraussetzungen für die einseitige Kündigung durch den Arbeitnehmer ableiten. Die Umstände, unter denen dem Arbeitnehmer ein Kündigungsrecht nach $\$ 38$ ArbVG zusteht, beziehen sich entweder auf eine grundlegende Vertragsverletzung (nicht rechtzeitige und vollständige Zahlung von Arbeitslöhnen und Sozialversicherungsbeiträgen) oder darauf, dass die Fortsetzung des Arbeitsvertrags wegen der Nichterfüllung oder unvollständigen Erfüllung von Pflichten (Zwangsarbeit durch Gewalt, Drohungen oder Einschränkungen der persönlichen Freiheit) unzumutbar ist. Hiervon ausgehend kann der belästigte Arbeitnehmer nur dann eine doppelte wirtschaftliche Entschädigung nach $\$ 87$ ArbVG verlangen, wenn die geduldete sexuelle Belästigung das geistige und seelische Wohlbefinden des belästigten Arbeitnehmers so stark beeinträchtigt, dass ihm die Fortsetzung der Arbeit für den Arbeitgeber nicht zugemutet werden kann.

\section{Fazit}

Regelungen gegen sexuelle Belästigung zielen nicht darauf, bestimmte Verhaltensweisen zu verbieten, sondern darauf, die sexuelle Selbstbestimmung jeder Person zu schützen. Dies soll auch für $\$ 1010$ ZGB gelten. Deshalb sollte „entgegen dem Willen des anderen“ im Sinne von $\mathbb{} 1010$ S. 1 ZGB als „unerwünscht“ interpretiert werden, was von einer opferbezogenen Sichtweise geprägt wird.

In Bezug auf die Haftung des Arbeitgebers wird von einem großen Teil der Literatur erwartet, die verschuldensunabhängige Haftung und verschuldensabhängige Haftung ins chinesische Recht zu übernehmen. Verkannt wird jedoch die fehlende Notwendigkeit und Durchführbarkeit einer solchen Einordnung. Der Schutz vor einer ungerechtfertigten Kündigung oder unangemessenen Versetzung ist schon im Rahmen des chinesischen Arbeitsrechts verwirklicht worden. Darum ist der Versuch unnötig, die mit der durch einen Vorgesetzten begangenen sexuellen Belästigung einhergehenden Kündigung oder Versetzung im Rahmen des Deliktsrecht zu erfassen. Fraglich ist nur, ob der betroffene Arbeitnehmer einen Anspruch gegen den Arbeitgeber auf Schadenersatz hat. Im Deliktsrecht haf-

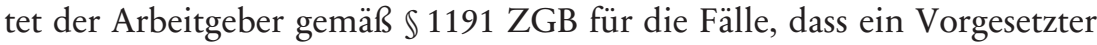
seine Untergebenen sexuell belästigt. Für sexuelle Belästigung durch gleichrangigen Kollegen greift $\$ 1198$ ZGB ein. Der Arbeitgeber haftet aber nur ergänzend. Der Arbeitnehmer kann nicht vom Arbeitgeber ver- 
langen, alle Schäden zu entschädigen. Selbst dann nicht, wenn der Arbeitgeber gar keine angemessenen Maßnahmen i. S. v. $\$ 1010$ ZGB ergriffen hat. Im Vertragsrecht sollte der Arbeitgeber grundsätzlich alle Schäden, einschließlich der immateriellen Schaden, ersetzen müssen. In der Rechtspraxis könnte sich ein Problem daraus ergeben, dass Gerichte dem Antrag auf immateriellen Schadenersatz wegen Vertragsverletzung nicht stattgegeben könnten. Langfristig gesehen ist zu erwarten, dass eine spezielle Vorschrift über die Haftungsbegründung und die Haftungsausfüllung im Rahmen der Haftung des Arbeitgebers in das ZGB eingeführt wird. 